\title{
Entstauungstherapie bei Lymphödem
}

\author{
anhand eines Patientenfalles (postoperatives Lymphödem Grad II des linken Beines \\ eines 64-jährigen Mannes nach Urothel-Carcinoma-in-situ mit Exstirpation von 7 links \\ inguinalen und 12 pelvinen Lymphknoten)
}

\section{Decongestive therapy for lymphedema}

\author{
based on a case report (postoperative lymphedema grade II of the left leg in a \\ 64-year-old male with carcinoma in situ of the urothelium following excision of 7 left \\ inguinal and 12 pelvic lymph nodes).
}

Autoren

M. Finkenrath

Institut

Hospital zum Heiligen Geist Kempen, Abteilung für

Phlebologie/Venenchirurgie

\section{Schlüsselwörter}

Lymphödem, Entstauungstherapie bei Lymphödem, Erhaltungstherapie bei Lymphödem, manuelle Lymphdrainage, intermittierende pneumatische Kompression, Kompressionsstrümpfe

Key words

Lymphedema, decongestant therapy, maintenance therapy, manual lymph drainage, intermittent pneumatic compression, compression stockings

eingereicht 23.07.2019

akzeptiert $\quad 09.08 .2019$

Bibliografie

DOI https://doi.org/10.1055/a-1004-6751

Phlebologie 2019; 48: 346-349

(c) Georg Thieme Verlag KG Stuttgart · New York ISSN 0939-978X

Korrespondenzadresse

Dr. Meike Finkenrath

Hospital zum Heiligen Geist Kempen, Abteilung für

Phlebologie/Venenchirurgie

Von-Broichhausen-Allee 1

47906 Kempen

E-Mail: meike.finkenrath@krankenhaus-kempen.de

\section{ZUSAMMENFASSUNG}

Anhand des nachfolgenden Fallberichtes soll gezeigt werden, welche Möglichkeiten in der Praxis bei einem häufig auftretendem lymphologischen Krankheitsbild, dem postoperativ nach Lymphknotenexstirpation entstandenen Lymphödem Grad II der Beine durchführbar sind und welche Leiden dieses Krankheitsbild für die Patienten (bis hin zur Immobilität) mit sich bringt. Der Ablauf von der Diagnostik (Eruieren der Ursache) über die Einleitung der initial standardisierten Therapie soll erläutert werden. Im weiteren Verlauf muss diese Therapie - auch das soll dargestellt werden - regelmäßig individuell angepasst werden. Darüber hinaus wird gezeigt, wie wichtig die Patientenaufklärung und -compliance bei diesem Krankheitsbild sind.

\section{ABSTRACT}

Based on the following case report it is shown which therapeutic measures can be utilized in daily practice to deal with a common lymphatic disorder: the postoperative lymphedema of the leg after excision groin or pelvic lymph nodes. The suffering associated with this condition, which can even lead to immobilization of the patients, is demonstrated. The therapeutic approach from diagnosis to standardized treatment is outlined, as well as the changes that are necessary during the individual course of the therapy. In addition, it is shown how important patient information and compliance are in order to reach a good therapeutic result. 


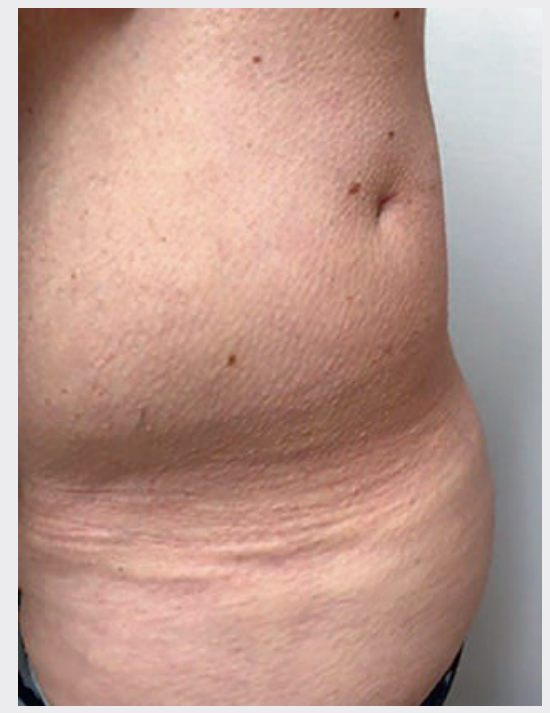

-Abb. 1 Lymphödem, Ausgangsbefund.

Nach der Definition des Lymphödems in der aktuellen AWMF-Leitlinie [1] ist es eine „... im unbehandelten Zustand, progrediente, chronische Erkrankung als Folge einer primären oder sekundären Schädigung des Lymphdrainagesystems (Lymphkapillaren, Lymphkollektoren, Lymphknoten, Lymphstämme) mit konsekutiver Vermehrung und Veränderung der interstitiellen Gewebsflüssigkeit. Im weiteren Verlauf ist die Erkrankung durch eine Alteration von Geweben, mit einer Zunahme von Binde- und Fettgewebe sowie Veränderung der extrazellulären Matrix (Hyaluronsäure, Kollagen, Glykosamine) gekennzeichnet.“

So stellte sich Mitte Februar dieses Jahres ein athletischer 64-jähriger Mann in unserer Sprechstunde vor und berichtete, vor nicht ganz einem Jahr (11 Monaten) aufgrund eines Urothel-Carcinomain-situ mittels Zystoprostatektomie und Anlage einer lleum-Neoblase operiert worden zu sein. Dabei sei eine bi-pelvine Lymphadenektomie (links > rechts) und eine Harnleiterresektion durchgeführt worden. Nachfolgend habe sich eine Lymphozele links inguinal gebildet, welche gefenstert worden sei. Während des Jahresverlaufes habe er nahezu seine alte Konstitution zurückgewonnen; nur das linke Bein sei zunehmend angeschwollen und nun habe sich auch das Bindegewebe verhärtet, sodass ein normales Gehen nicht möglich sei. Die Knickbewegung im Kniegelenk sei aufgrund des ,enger gewordenen Bindegewebes “ eingeschränkt; dabei wünsche er sich als leidenschaftlicher Radfahrer nur, dass das Radfahren wieder möglich sei. Bisher keine Behandlung, außer Tragen von rundgestrickten Kompressionsstrümpfen KKL II A-G ohne Fußspitze, offen.

Bei der klinischen Untersuchung zeigte sich folgendes Bild ( $\triangleright$ Abb. 1). Ohne Längendifferenz fand sich die Ödemkonsistenz derb fibrotisch, eine Dellenbildung bleibend, Stemmer-Zeichen mit Gewebefibrosierung positiv, insgesamt war das Ödem eher Hüft-/Oberschenkel-betont (s. Foto); Lymphknoten konnten sonographisch links inguinal nicht nachgewiesen werden. Puls palpabel. Funktionseinschränkung der Beugung im linken Kniegelenk auf 0-0-30 Grad. Hautbefund blande. Es wurde ein sekundäres Lymphödem des linken Beines, Stadium II, diagnostiziert, da sich

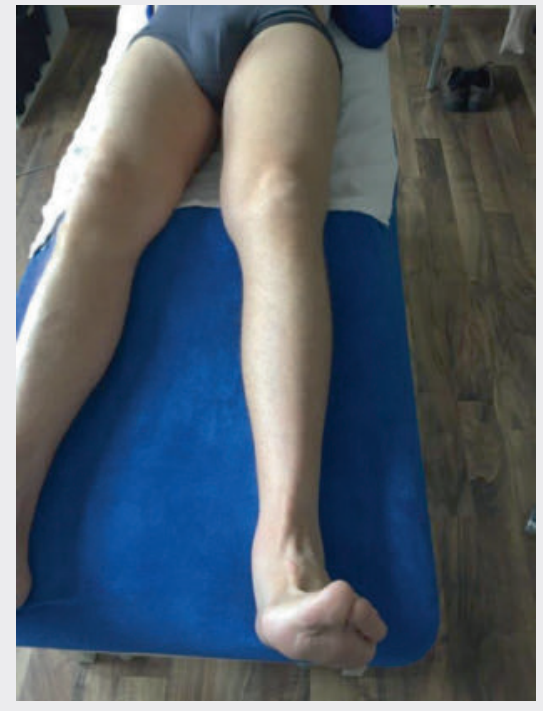

Abb. 2 Befund nach erster MLD.

bereits Gewebefibrosierungen zeigten ( $\mathbf{A} \mathbf{A b} \mathbf{b} . \mathbf{1})$. Ein Hochlagern des Beines war ohne Wirkung.

Eine Insuffizienz im epifaszialen oder tiefen Beinvenensystem konnte duplexsonographisch ausgeschlossen werden. Als Therapieziel vereinbarten wir eine Reduktion des Ödems, sodass ein Radfahren wieder möglich sein sollte; der Patient war hochmotiviert und wurde über Hautpflege zur Verhinderung des Erysipels etc. aufgeklärt. Er selber berichtete von einer maschinellen Lymphdrainage als Heimgerät, von der er gehört habe. Wir einigten uns initial zu einer manuellen Lymphdrainage (MLD, > Abb. 2), 6×45 Minuten für das linke Bein mit anschließender Kompressionsbandagierung mit einer Schaumstoffwickel unter einer Kurzzugwickel sowie Übungsbehandlungen in der Bandagierung (medizinisch komprimierende Bandagen mit hohem Arbeitsdruck, > Abb. 3).

Nach der fünften manuellen Lymphdrainage sollten noch im Liegen flachgestrickte Kompressionsstrümpfe der KKL II A-G angemessen werden ( $\triangleright$ Abb.4). Zudem sollte er einen Antrag auf eine Heilmittelverordnung außerhalb des Regelfalls (s. Genehmigungsverfahren zur Heilmittelverordnung außerhalb des Regelfalls) zur kontinuierlichen manuellen Lymphdrainage aufgrund der Tumoreigenanamnese beantragen. Einen Monat später stellte sich der Patient erneut vor. Weiterhin ungeduldig ob seiner doch schneller erwarteten besseren Beweglichkeit bittet er um Erweiterung der Therapiemaßnahmen. Das Ödem zeigt sich palpatorisch deutlich weicher. Wir empfahlen aufgrund der ambulant beinahe ausgereizten Maßnahmen (eine $2 \times \mathrm{tgl}$. ambulante manuelle Lymphdrainage mit Kompressionsbandagierung und Übungsbehandlung in der Bandagierung über die Dauer von mind. 24 Tagen im Sinne der Phase I der komplexen physikalischen Entstauungstherapie konnte nicht durchgeführt werden, da er keinen Lymphtherapeuten fand, der dazu bereit war) zeitnah einen lymphologischen Reha-Aufenthalt in einer Lymphfachklinik, welchen der Patient auch gleich beantragte. Zudem empfahlen wir ein maschinelles 12-Kammer-Lymphdrainage-Gerät mit Bauchteil (AIK, apparative intermittierende Kompression) zur Heimbehandlung, um die Abstän- 


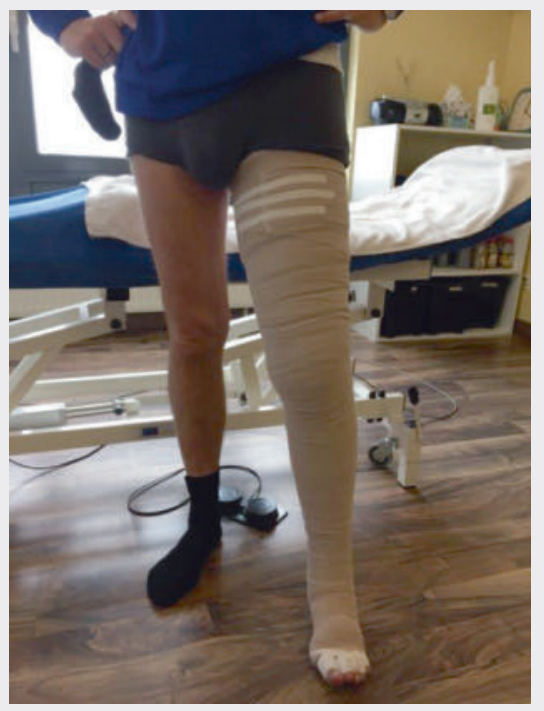

-Abb. 3 Mehrlagige Kompressionsbandagierung.

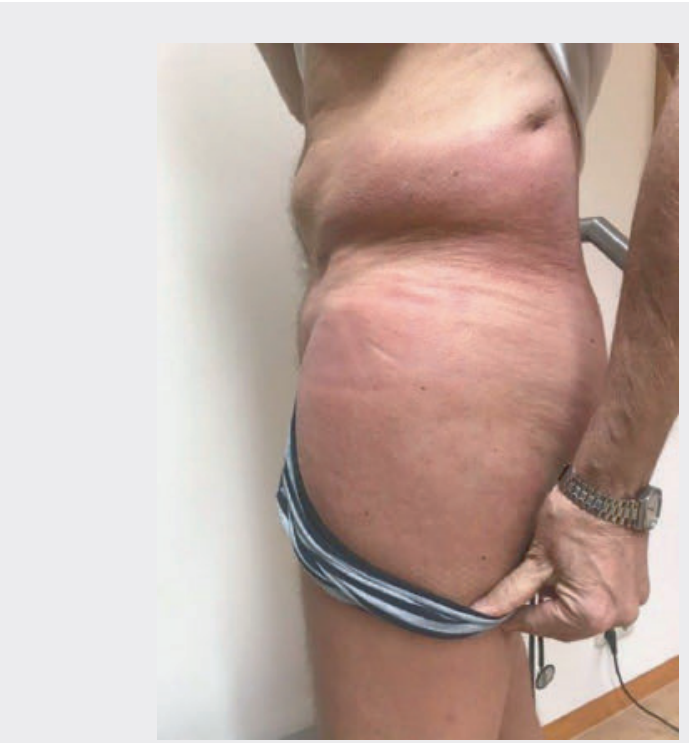

- Abb. 5 Hüftbetontes akutes Erysipel über Hautinfekt im Bereich des ehemaligen Fistelkatheters nach „exzessiver“ Anwendung der AIK (ohne vorherige Schulung über Selbstanregung des Lymphtransports).

de zwischen den derzeitig stattfindenden ambulanten manuellen Lymphdrainage-Terminen zu überbrücken. Der Patient bekam unverzüglich ein derartiges Leihgerät aus einem Sanitätshaus zur Verfügung gestellt.

Einen Monat später stellte er sich hochfieberhaft in reduziertem AZ und depressiver Verstimmung mit einem akuten Erysipel des linken Beines und Beckens notfallmäßig zur stationären Aufnahme vor ( $\mathbf{A b b} \mathbf{5}$ und $\triangleright$ Abb. $\mathbf{6}$ ). Er hatte in den vergangenen Wochen hochmotiviert - leider ohne adäquate Anleitung und vorherige Schulung über die Anregung der Lymphbahnen - das ma-

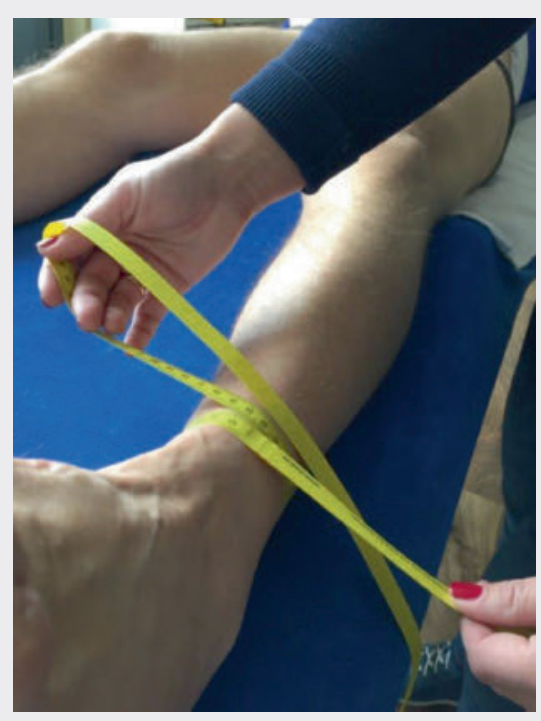

-Abb. 4 Ausmessen flachgestrickter MKS im Liegen.

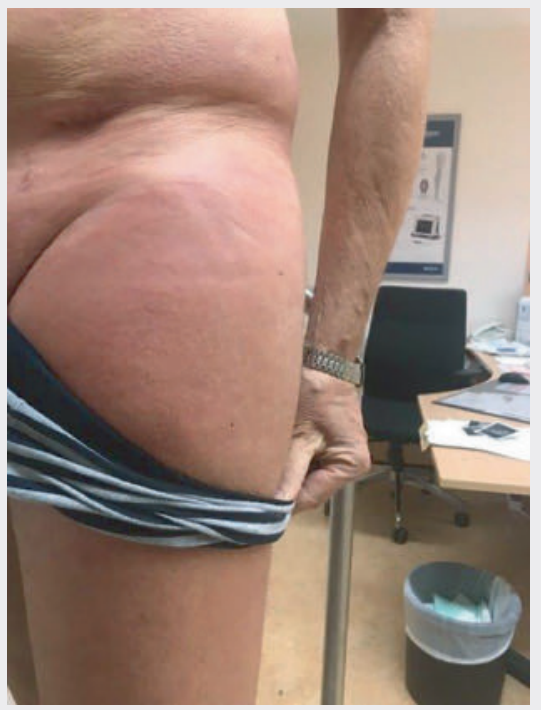

- Abb. 6 Hüftbetontes akutes Erysipel über Hautinfekt im Bereich des ehemaligen Fistelkatheters nach „exzessiver“ Anwendung der AIK (ohne vorherige Schulung über Selbstanregung des Lymphtransports).

schinelle Lymphdrainagegerät mehrmals täglich „exzessiv“ angewendet und durch einen Hautinfekt im Bereich des vormals angelegten Fistelkatheters hat sich ein hüft- und oberschenkelbetontes Erysipel ausgebildet.

Nach adäquater Antibiotikatherapie und supportiven Maßnahmen sowie einer erneuten ausführlichen Aufklärung über Ursache und die Langfristigkeit einer lymphologischen Behandlung, konnte der Patient rasch entlassen werden. Noch unter stationären Bedingungen (nach Rückgang der klinischen sowie laborchemischen Infektparameter) wurde die manuelle Lymphdrainage sowie das 
- Tab. 1 Messdaten des Patienten.

\begin{tabular}{|c|c|c|c|c|}
\hline Tag & Tageszeit & Oberschenkel & Unterschenkel & Fuß \\
\hline \multirow[t]{2}{*}{1} & Morgens & 53 & 35 & 27 \\
\hline & Abends & 52 & 35 & 26 \\
\hline \multirow[t]{2}{*}{2} & Morgens & 52 & 34 & 25 \\
\hline & Abends & 54 & 36 & 26 \\
\hline \multirow[t]{2}{*}{3} & Morgens & 51 & 31 & 25 \\
\hline & Abends & 53 & 36 & 26 \\
\hline \multirow[t]{2}{*}{4} & Morgens & 52 & 33 & 27 \\
\hline & Abends & 53 & 36 & 26 \\
\hline \multirow[t]{2}{*}{5} & Morgens & 52 & 36 & 26 \\
\hline & Abends & 53 & 36 & 26 \\
\hline \multirow[t]{2}{*}{6} & Morgens & 52,5 & 35,5 & 26,5 \\
\hline & Abends & 53 & 37 & 26 \\
\hline \multirow[t]{2}{*}{7} & Morgens & 53 & 34 & 26 \\
\hline & Abends & 53 & 36 & 26 \\
\hline \multirow[t]{2}{*}{8} & Morgens & 52,5 & 36 & 26 \\
\hline & Abends & 53 & 36 & 24 \\
\hline \multirow[t]{2}{*}{9} & Morgens & 52 & 36 & 22 \\
\hline & Abends & 54 & 37 & 23 \\
\hline \multirow[t]{2}{*}{10} & Morgens & 52,5 & 35,5 & 26 \\
\hline & Abends & 52 & 37,5 & 26 \\
\hline \multirow[t]{2}{*}{11} & Morgens & 52 & 34 & 25 \\
\hline & Abends & 52,5 & 35 & 26 \\
\hline \multirow[t]{2}{*}{12} & Morgens & 53 & 35 & 26 \\
\hline & Abends & 53 & 35 & 26 \\
\hline \multirow[t]{2}{*}{13} & Morgens & 52 & 35,5 & 26 \\
\hline & Abends & 54 & 36 & 26 \\
\hline 15 & Morgens & 52,5 & 34 & 25 \\
\hline 17 & Morgens & 52,5 & 35 & 26 \\
\hline \multirow[t]{2}{*}{19} & Morgens & 52 & 35 & 26 \\
\hline & Abends & 53 & 35 & 26 \\
\hline 21 & Morgens & 53 & 35 & 26 \\
\hline \multirow[t]{2}{*}{22} & Morgens & 54 & 35,5 & 27 \\
\hline & Abends & 54 & 36 & 26 \\
\hline \multirow[t]{2}{*}{23} & Morgens & 53 & 35,5 & 26 \\
\hline & Abends & 53 & 34 & 26 \\
\hline \multirow[t]{2}{*}{24} & Morgens & 53 & 34 & 26 \\
\hline & Abends & 54 & 33 & 27 \\
\hline 25 & Morgens & 55,5 & 34,5 & 26 \\
\hline 26 & Morgens & 52 & 33,5 & 25 \\
\hline 27 & Morgens & 54 & 36,5 & 26 \\
\hline 28 & Morgens & 55 & 35 & 26 \\
\hline
\end{tabular}

Tragen von flachgestrickten Kompressionsstrümpfen wieder aufgenommen.

Es ergab sich obiges Bild ( $\bullet$ Abb. 7) und auch die selbst gemessenen Umfangsmaße zeigten sich regredient $(\triangleright$ Tab. 8). Ambulant erhielt er $2-3 \times /$ Woche manuelle Lymphdrainage à 60 Minuten für das linke Bein, ergänzte dies durch das $1 \times$ tgl. Anwenden der ma-

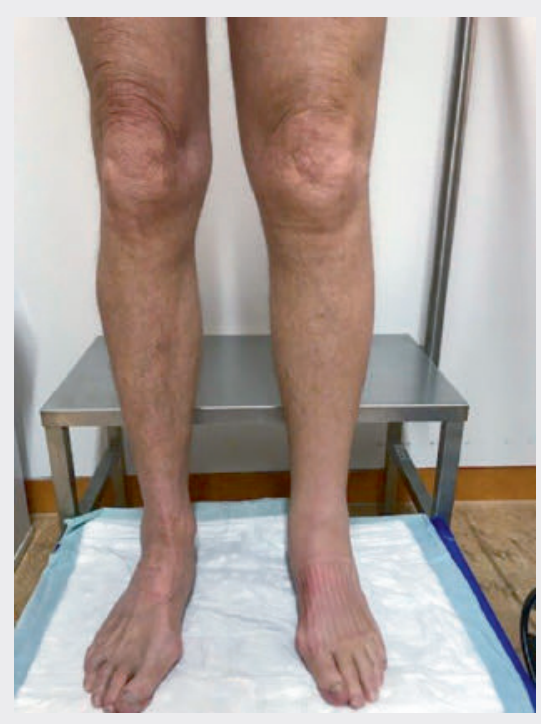

-Abb.7 Ambulante, derzeitige Erhaltungstherapie.

schinellen Lymphdrainage (derzeit ohne Steigerung des Anpressdruckes) nach Selbstanregung der Lymphbahnen und trug täglich den Kompressionsstrumpf, in dem er selbst moderate Bewegung (Spazieren, Bewegungstherapie im Wasser etc.) durchführte. Der Patient ist soeben in den Urlaub gefahren und kann sich deutlich besser bewegen; auch vorsichtiges Radfahren ist möglich. Somit hat dieser Patient - obwohl er noch immer auf die Zusage zur lymphologischen Reha wartet - seine Therapieziele (Verbesserung des Lymphabflusses, Erweichung fibrosklerotischer Gewebsveränderungen, Verbesserung der Funktionsdefizite, Verbesserung der Lebensqualität) erreicht.

Abschließend zeigt sich, dass das stadiengerechte Abstimmen der komplexen physikalischen Entstauungstherapie (Entstauungs-, Optimierungs- und Konservierungsphase) einer adäquaten Schulung und Aufklärung des Patienten (u. a. der Selbstbehandlungsmöglichkeiten und -einschränkungen) und der die Therapie zu überwachenden Lymphtherapeuten (Schulungsauftrag) und Ärzte bedarf und aufgrund des Zeitaufwandes schwerlich unter ambulanten Bedingungen adäquat umgesetzt werden kann. Hier ist ggf. eine spezielle Ausbildung des medizinischen Assistenzpersonals hilfreich für das Zeitmanagement im Handling mit dieser Patientengruppe.

\section{Interessenkonflikt}

Die Autoren erklären, dass kein Interessenkonflikt besteht.

\section{Literatur}

[1] AWMF online-Leitlinien Lymphologie:Gliedmaßenlymphödeme; http://www.uni-duesseldorf.de/AWMF/II/058-001.htm 